\title{
Improving Saw Reliability in Cutting a Moving Pipe
}

\author{
V. S. Maslov, Yu. B. Chechulin, and S. K. Buinachev \\ Yeltsin Ural Federal University, Yekaterinburg, Russia
}

\begin{abstract}
The saws used to cut moving pipe are studied. The basic cutting parameters are determined, and improvement in saw performance is discussed.
\end{abstract}

DOI: $10.3103 / \mathrm{S} 0967091213050112$

We need better information regarding the highspeed cutting of pipe by disk saws, when the pipe is in continuous motion. In such systems, to ensure optimal energy expenditure and performance of the equipment and also optimal product quality, the supply conditions for disk saws turning at azimuthal speeds greater than $100 \mathrm{~m} / \mathrm{s}$ must be consistent with the linear velocity of the blank. Accordingly, in the present work, we study the operation of mobile saw systems with linear motion of the pipe platform and saw carriage and with a planetary drive for plane-parallel motion of the disk saw.

We consider two main designs of the saw system: a carriage-type system and a planetary system. In the carriage-type system (Fig. 1), the disk saw (with drive 1 and clamp 2) is attached to carriage 6 , which is moved by platform 3 in the course of cutting, with the same speed and direction as pipe 4 . The disk and clamp are moved in the direction of the pipe by means of a supply device 5 (for instance, a hydraulic cylinder). Thus, plane-parallel motion of the disk is synchronized with the pipe motion. The speed of platform 3 is specified by a signal from sensors determining the pipe speed. The speed of carriage 6 is regulated.

In a planetary system, conical pairs with unit gear ratio ensure plane-parallel motion of the disk. A sensor system synchronizes the plane-parallel motion of the disk with the linear motion of the pipe blank. The disk has an autonomous motor and is perpendicular to the pipe. Two pairs of conical gears ensure a constant angle of $90^{\circ}$ with complete rotation of the saw's cantilever arm.

Both types conform to the fundamental configuration in Fig. 2. The equipment employed in such systems requires improvement to eliminate certain problems. For example, variation in the tensile force interferes with belt performance in the carriage-type system. Excess tensile force in the belt reduces the fault-free operating time of the motor and spindle. The clamp in the planetary system does not ensure uniform support of the pipe in cutting. Nonuniform support results in an unpredictable cutting trajectory and potentially to accidents. The intermediate roller conveyer at the output of the planetary system is too slow: the large moving masses prevent satisfactory operating speeds and may again lead to accidents. The transfer system at the output of the planetary system must ensure safe motion of the measured pipe sections and rapid removal of the offcuts from the line. However, the existing design, with smooth rollers, does not permit deflection of the pipe on the conveyer. The operational reliability of the lateral push rods for the offcuts is poor.

It is difficult to determine the cutting parameters in the system on account of the high cutting speeds and

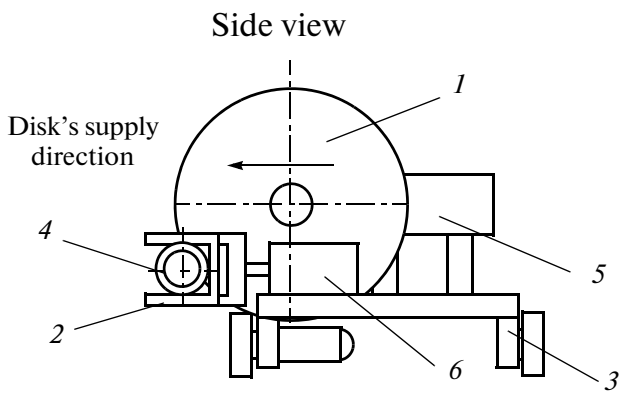

Plan view

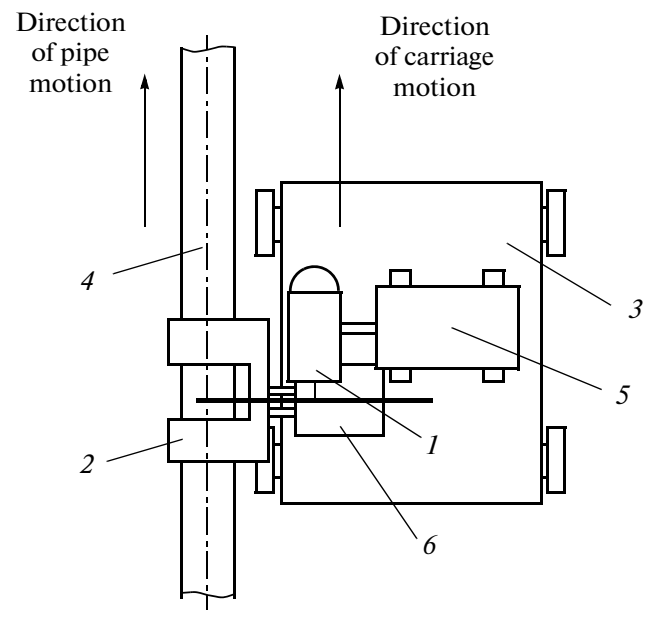

Fig. 1. Carriage-type system. 


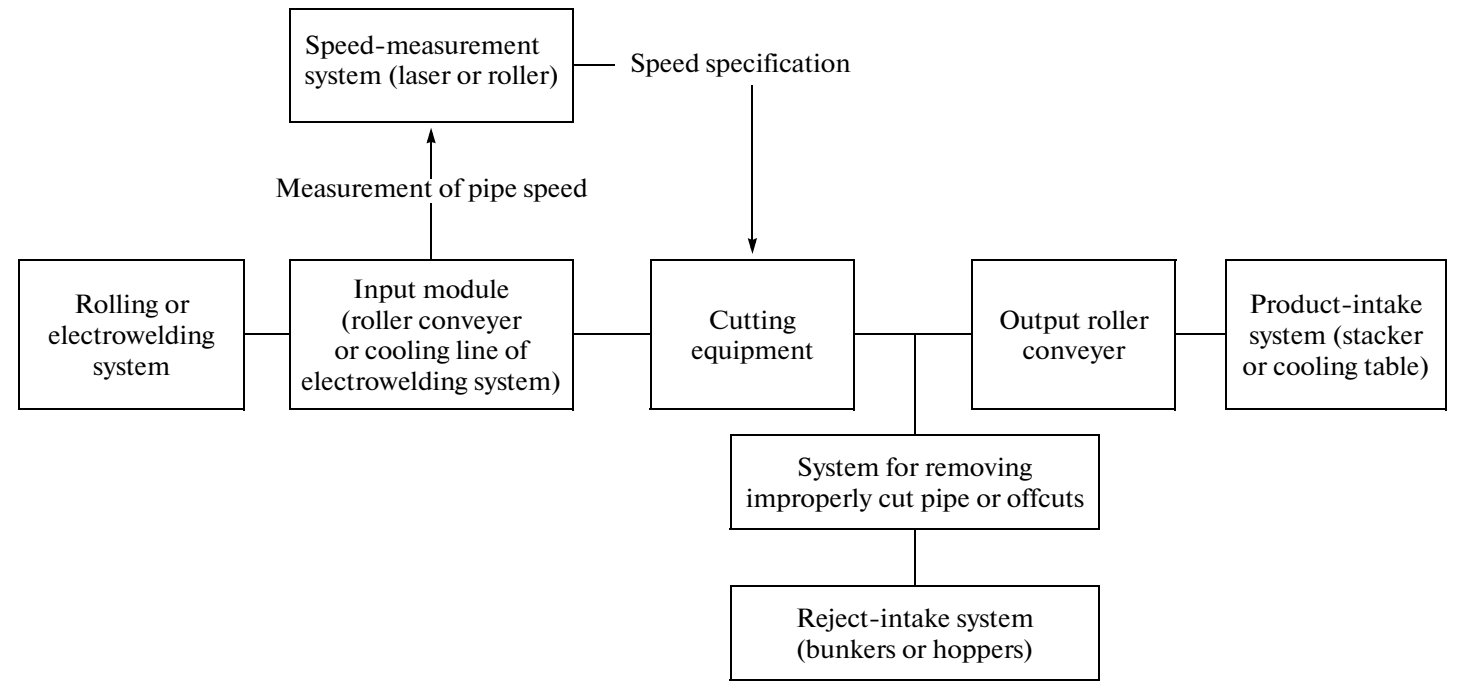

Fig. 2. Configuration of cutting systems.

complex kinematics. Such cutting systems require further study. To determine the primary cutting parameters (the cut width and depth, supply speed, and supply per saw tooth), we must first adequately describe the kinematics of the process with any relative positions of the disk and pipe blank. The cutting processes in such systems are distinctive in the following respects:

(1) The geometry of the cut part. Different numbers of saw teeth are operational in different intervals, with a maximum at the walls and a minimum in the pipe's inner cavity;

(2) High rotary speed of the saw with relatively slow supply. That results in extremely small cutting depth (hundredths of a mm). Consequently, the cutting process resembles plastic deformation of the metal;

(3) Different cutting forces on each saw tooth. That is associated with nonuniform supply motion of the disk.

The cutting depth $S$ at each time $t$ may be determined by the following formula for a carriage-type system [2]

$$
S=f\left(t, r_{\mathrm{d}}, r_{\mathrm{pi}}, L_{\mathrm{w}}, \theta, \omega_{\mathrm{d}}, H, n\right),
$$

where $r_{\mathrm{d}}$ and $r_{\mathrm{pi}}$ are the disk and pipe radii, respectively; $L_{\mathrm{w}}$ is the working length of the equipment; $\theta$ is the angular spacing of the saw teeth on the disk; $\omega_{\mathrm{d}}$ is the speed of the saw disk; $H$ is the difference in height of the center of the disk and the center of the pipe; $n$ is the number of saw teeth that are simultaneously in operation.

Correspondingly, for the planetary system

$$
S=f\left(t, r_{\mathrm{d}}, r_{\mathrm{pi}}, V_{\mathrm{pi}}, L, R, \theta, \omega_{\mathrm{d}}, H, n\right),
$$

where $V_{\mathrm{pi}}$ is the speed of the pipe; $L$ is the distance from the axis of the system's column to the pipe axis; $R$ is the system's cantilever length.
On the basis of these formulas, we may derive the dependence of the cutting force $F$ on the cutting time $t$ by means of the approach in [1]

$$
F=f\left(t, S, \beta, \sigma_{\mathrm{st}}, n, B, \mu, \gamma, u, k_{c}, l_{\mathrm{re}}, F_{\mathrm{sh}}\right),
$$

where $\beta$ is the Lode coefficient; $B$ is the width of the saw disk; $n$ is the number of saw teeth that are simultaneously in operation; $\mu$ is the frictional coefficient; $\gamma$ is the tooth's front angle; the coefficient $u$ depends on the front angle ( $u=1$ in the present case); $h_{1}$ is the thickness of the cut layer ( $h_{1}=S$ in the present case); $h_{2}$ is the chip thickness; $k_{c}=h_{2} / h_{1}$ is the chip-thickening coefficient; $\sigma_{\mathrm{st}}$ is the mean yield stress for the cut material at specified temperature, determined from the hardening graph for the given temperature on the basis of the calculated mean accumulated strain $e_{i}$ :

$$
e_{i}=1.155\left(1+e^{-\cos \gamma}\right) \cos \gamma,
$$

$F_{\text {sh }}$ is the force on the disk's lateral surfaces

$$
F_{\mathrm{sh}}(t)=0.5 \beta \sigma_{s} s_{\mathrm{sh}},
$$

$s_{\mathrm{sh}}$ is the area of the lateral shear surface in cutting (determined as a function of the time); $l_{\mathrm{re}}=\frac{h_{3}}{\tan \alpha}$ is the contact length of the tooth's rear surface with the pipe blank; $h_{\mathrm{re}}$ is the height of the elastic-strain zone at the tooth's cutting edge in contact with its rear surface.

As an example, we plot the calculated cutting forces for a carriage-type system and a planetary system in Figs. 3 and 4, respectively.

In experiments, we cut a stationary pipe at supply speeds typically seen in the cutting of moving pipe, so as to eliminate interference. We record the motor cur-

\footnotetext{
${ }^{1}$ In the case of hot cutting, weakening is observed on account of shift of the strengthening curve toward the minimum.
} 


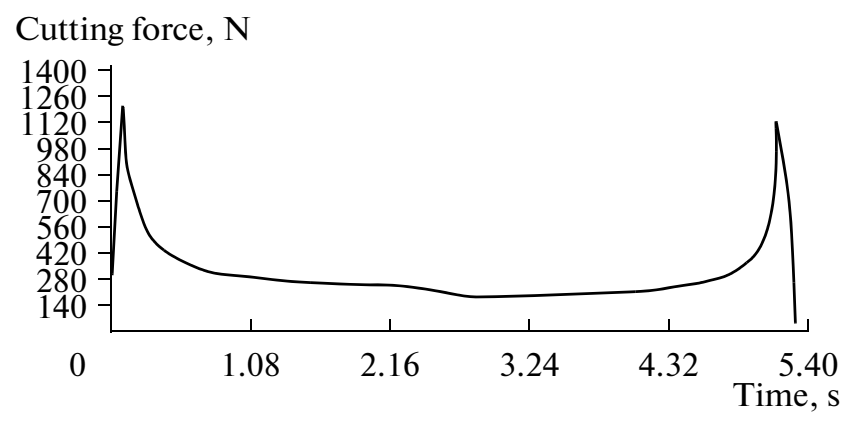

Fig. 3. Time dependence of the calculated cutting forces for a carriage-type system $(325 \times 6 \mathrm{~mm}$ pipe $)$.

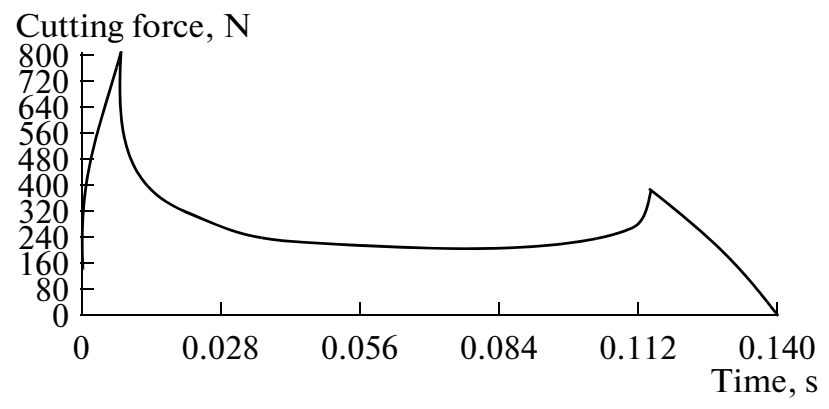

Fig. 4. Time dependence of the calculated cutting forces for a planetary system $(89 \times 8 \mathrm{~mm}$ pipe $)$.

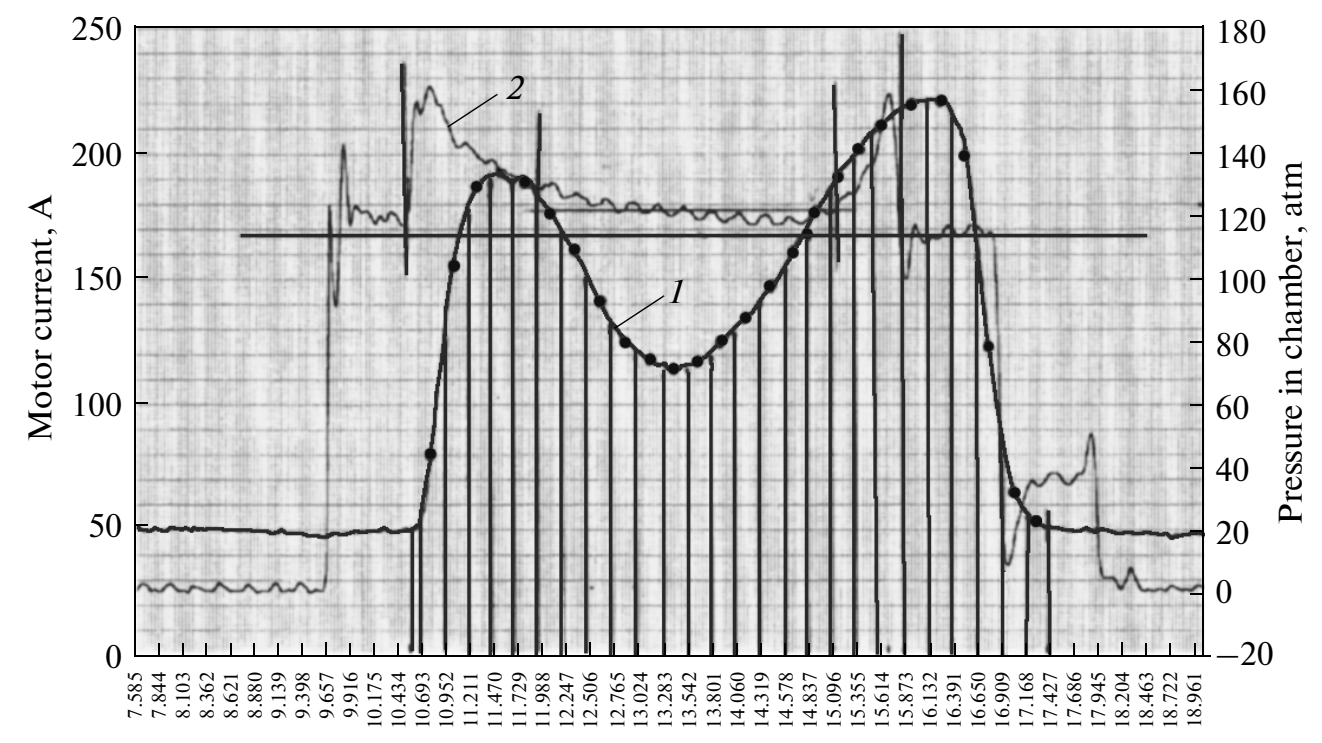

Time, s

Fig. 5. Experimental dependence of the motor current (1) and the pressure in the chamber of the hydraulic cylinder (2) when cutting $325 \times 6 \mathrm{~mm}$ pipe.

rent in one phase and the pressure in the chamber of the hydraulic cylinder used for disk supply (Fig. 5).

To assess the dynamic behavior of the system we have written a program on the basis of Python software. The motion is described by means of the following generalized coordinates: $\theta_{d}$, the coordinate of the motor's rotor; $\vartheta_{s}$, the coordinate of the driven pulley in the belt transmission; and $\vartheta_{f}$, the coordinate of the saw disk.

The equation of motion then takes the form

$$
A \ddot{\vartheta}+B \dot{\vartheta}+C \vartheta+D=0,
$$

where $A$ is the matrix of moments of inertia; $B$ is the matrix of reduced dissipation coefficients; $C$ is the matrix of reduced rigidity coefficients; $D$ is the vector of reduced external forces.

Using the mathematical model, we determine the speed, torque, motor voltage, and motor power as a function of the time. In Fig. 6, we plot the torque $M_{\text {to }}$ and cutting force $F$ as a function of the time. In this case, the torque reproduces the variation of the motor current. These curves correspond to the experimental data for the torque and cutting force. Comparison shows that the energy balances over the cycle are in good agreement for the theoretical and experimental results.

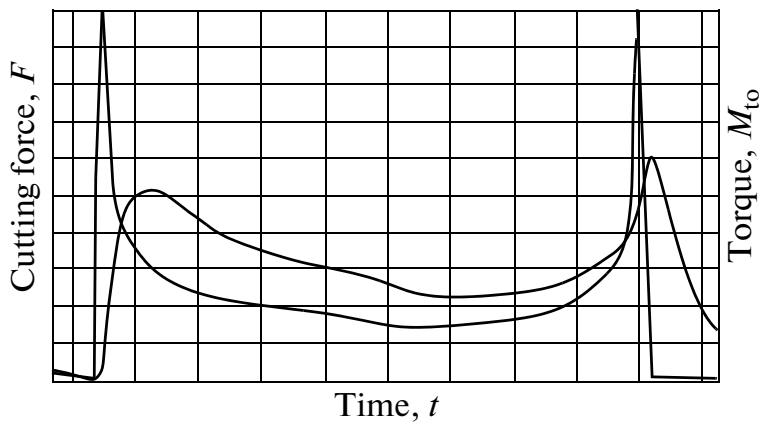

Fig. 6. Time dependences of the torque $M_{\text {to }}$ and the cutting force $F$. 


\section{CONCLUSIONS}

By the proposed approach, we may calculate the forces and dynamic loads in pipe cutting, taking account of the pipe and tool geometry, the speeds, and the supply as a function of the time. On the basis of the calculated and experimental load data, the best tension may be found for the belt transmission in the carriage-type system, so as to avoid periodic downtime. In addition, the theoretical kinematic data and cutting forces for the planetary system provide the basis for modernization of the equipment in the pipe-cutting line [3]: a new system for pipe support in the course of cutting [4]; a new intermediate roller conveyer with improved speed and reliability in discarding short offcuts [5]; and a system for clearing long offcuts from the line of the saw.

\section{REFERENCES}

1. Vorontsov, A.L., Sultan-zade, N.M., and Albagachiev, A.Yu., Development of a new theory of cutting, Russ. Eng. Res., 2008, nos. 1-12.

2. Chechulin, Yu.B., Maslov, V.S., and Buinachev, S.K., Determining the parameters of high-speed cutting of moving pipe, Stal', 2012, no. 9, pp. 84-88.

3. Chechulin, Yu.B., Maslov, V.S., Vert, V.Yu., and Treskin, V.V., Modernization of saw systems for safe cutting of moving pipe, Stal', 2012, no. 10, pp. 69-72.

4. Chechulin, Yu.B., Maslov, V.S., and Panfilov, A.Yu., Russian Patent 2389572, Byull. Izobret., 2011, no. 14.

5. Chechulin, Yu.B., Treskin, V.V., and Maslov, V.S., Russian Patent 111038, Byull. Izobret., 2011, no. 34.

Translated by B. Gilbert 